\title{
Physical properties of the dust in the Solar System and its interrelation with small bodies
}

\author{
I. Mann ${ }^{1}$, A. Czechowski ${ }^{2}$, H. Kimura ${ }^{3}$, M. Köhler ${ }^{1}$, \\ T. Minato ${ }^{1}$ and T. Yamamoto ${ }^{3}$ \\ ${ }^{1}$ Institut für Planetologie, Wilhelm-Klemm-Str. 10, 49149 Münster, Germany \\ email: imann@uni-muenster.de \\ ${ }^{2}$ Space Research Center, Polish Academy of Sciences, Warsaw, Poland \\ email: ace@cbk.waw.pl \\ ${ }^{3}$ Institute of Low Temperature Science, Hokkaido University, Sapporo 060-819, Japan
}

\begin{abstract}
Dust particles in the solar system are produced from the small bodies: asteroids, comets, meteoroids and Kuiper belt objects. A further source of dust is provided by the warm interstellar medium that the Sun is currently embedded in and that streams into the solar system. We review the physical properties of solar system dust and trace back its interrelation with the small solar system bodies. Comets contain relatively pristine material that they transport to the inner solar system. The alteration of dust in the vicinity of comets is complex and connected to the gas evolution, but a significant part of the organic dust material survives these alterations. The optical properties of cometary dust are best described with a mixture of silicate and carbon bearing materials. As far as the darkness of the cometary material is concerned, according to recent models, this is not a result of the porosity, but rather of the darkness of the carbon bearing component. This does not contradict the observation of silicate features in the thermal emission brightness of cometary dust, since porous mixtures of silicate and carbon bearing dust can produce the observed polarization and albedo characteristics, as well as the silicate features. The carbon-bearing component is most likely an organic refactory component. The relative contributions of different sources change within the solar system dust cloud and depend as well on the measurement technique considered. In particular, the dust from asteroids, which provides a large component of the dust near Earth orbit, is also preferably seen with most of the detection methods. The majority of dust inward from $1 \mathrm{AU}$ is produced from cometary dust and meteoroids. Dust material evaporation induced by collisions inward from 1 AU produces a minor heavy ion component in the solar wind plasma known as inner source pick-up ions.
\end{abstract}

Keywords. comets: general, interplanetary medium, meteors, meteoroids

\section{Introduction}

The sources of dust in the solar system are the direct supply from asteroids by their collision fragmentation, the activity of comets and the collision fragmentation of meteoroids generated by these parent bodies. Dust particles are also produced by collision and erosion processes in the Kuiper belt and enter the solar system directly from interstellar space (see Figure 1). The activity of comets is driven by the heating and sublimation of volatiles of the cometary ice, which generates the dust seen in comae and tails of comets. Part of this dust is directly ejected in unbound orbits. The generation of larger cometary meteoroids is evident from the observation of cometary dust trails (see Figure 2). As we explain later in the text, we can assume that the majority of the solar system dust cloud near $1 \mathrm{AU}$ consists of material that has undergone collisional processes. 


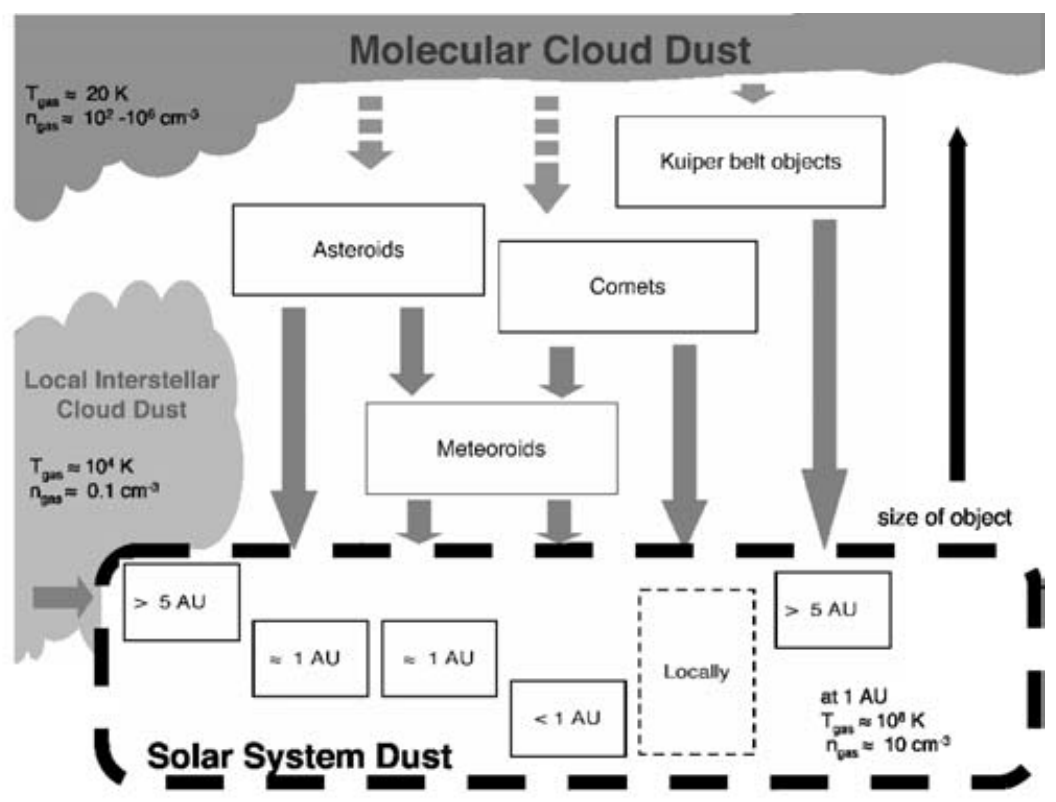

Figure 1. Dust sources in the solar system: the parent bodies of dust in the solar system are asteroids, comets and Kuiper belt objects, and interstellar dust particles directly enter the solar system from the local interstellar medium. The majority of dust outward from $5 \mathrm{AU}$ is interstellar dust and dust produced in the Kuiper belt. Dust near 1 AU comes from comets and asteroids. The majority of dust from asteroids and comets is not directly released from the parent bodies but generated from collisional fragmentation of meteoroids that come from these sources. The inner solar system dust cloud is replenished from collisions of asteroidal meteoroids.

Asteroids, comets and Kuiper Belt objects were formed from molecular cloud dust during solar system formation: The majority of the heavy elements beyond He existing in the interstellar medium (ISM) are condensed into solid dust particles. Dust particles are injected into the ISM after condensation in red giant stars, supernovae and novae. They grow by condensation, collisional accretion and coagulation while at the same time, the stellar UV radiation causes chemical reactions and in particular the formation of organic refractory material in icy condensates. Icy materials form in molecular cloud regions that also provide the conditions for solar system formation.

The properties of meteroids and dust particles reveal information about their parent bodies and about their different paths of evolution from the protoplanetary dust. The following discussion of the physical properties of dust in the solar system will concentrate on the processing of the cometary dust material. This provides a relatively pristine sample of material that can be studied by various methods. We will first discuss the dust properties derived from observations of the solar system dust cloud. The next section describes observational results about the dust processing in the vicinity of comets, followed by a discussion of the cometary dust properties. We then discuss the contribution of different parent bodies to the dust cloud, and finally discuss the demise of dust in the vicinity of the Sun and interactions with the solar wind.

\section{Dust properties derived from different observations}

Observational data about dust in the solar system are obtained from the observation of the Zodiacal ligh and the F-corona, the part of the corona that is produced by 


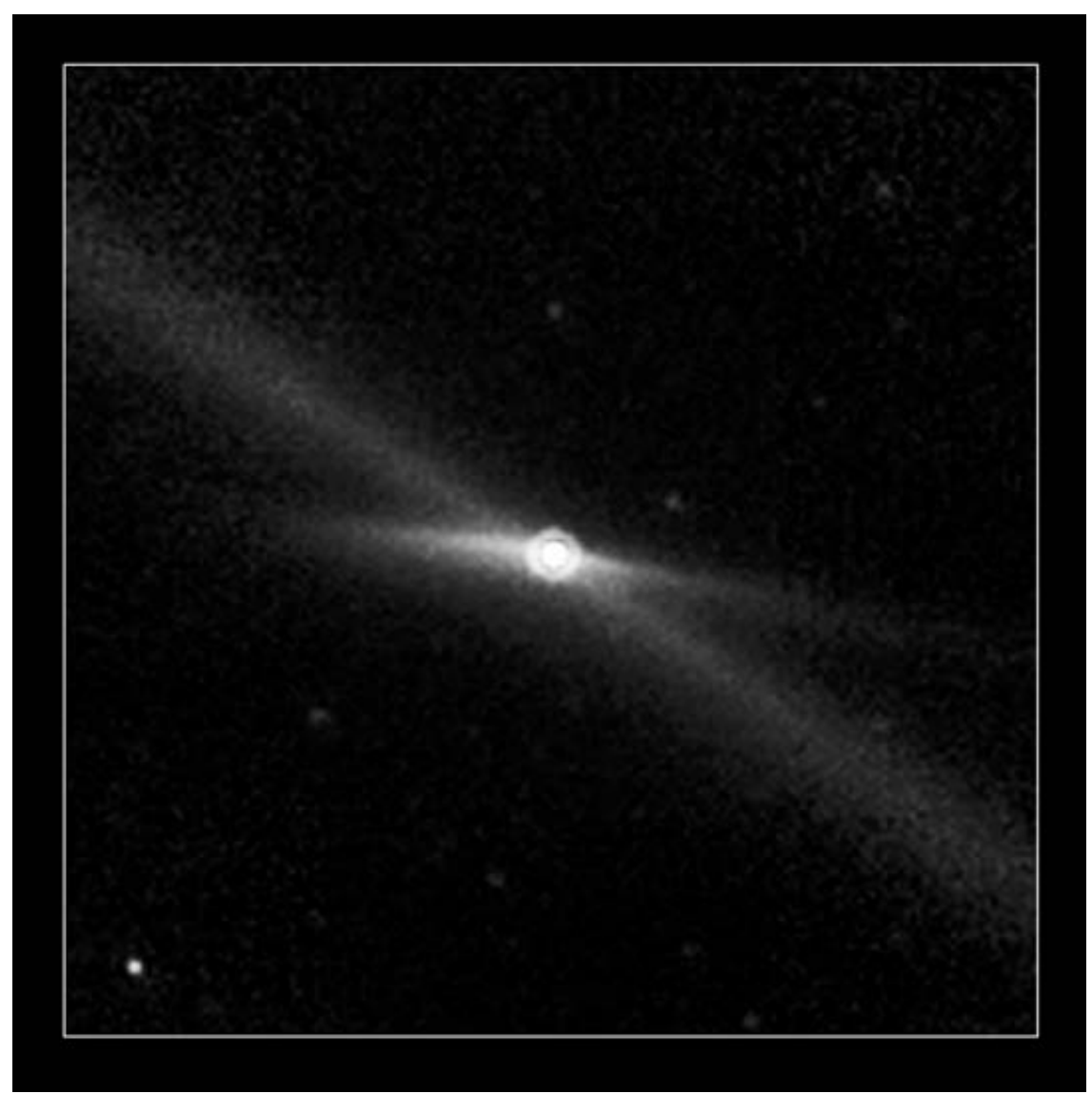

Figure 2. The dust trail of Comet Encke at 2 AU observed from Spitzer Space Observatory, image NASA/JPL-Caltech/M. Kelley (Univ. of Minnesota).

interplanetary dust, and from meteor observations of particles entering Earth's atmosphere. Laboratory studies are possible for collected samples of dust and micrometeorites. In-situ measurements of dust in the interplanetary medium are carried out from spacecraft. Data about cometary dust are obtained from observations of cometary comae, dust tails and dust trails as well as from spacecraft measurements during encounters with a comet.

\subsection{Zodiacal Light and F-corona}

The Zodiacal light (see Figure 3) and Zodiacal emission brightness data predominantly describe particles in the 1 to $100 \mu \mathrm{m}$ size range at distances from about 0.7 to $1.3 \mathrm{AU}$ close to the ecliptic plane (Levasseur-Regourd, Mann, Dumont et al. 2001). The overall distribution of dust number densities in the Zodiacal cloud can be explained with particles in low eccentricity and low inclination orbits that drift toward the Sun due to deceleration by the Poynting-Robertson effect (Mann 1998). The Zodiacal light smoothly continues into the solar F-corona brightness, but derived average optical properties are inconsistent with a gradual change of particles properties at distances smaller than about 0.5 AU from the Sun: Explaining the data requires a change of the size distribution of dust, a change of the dust cloud composition with distance from the Sun or a combination of both (Mann 1998). 


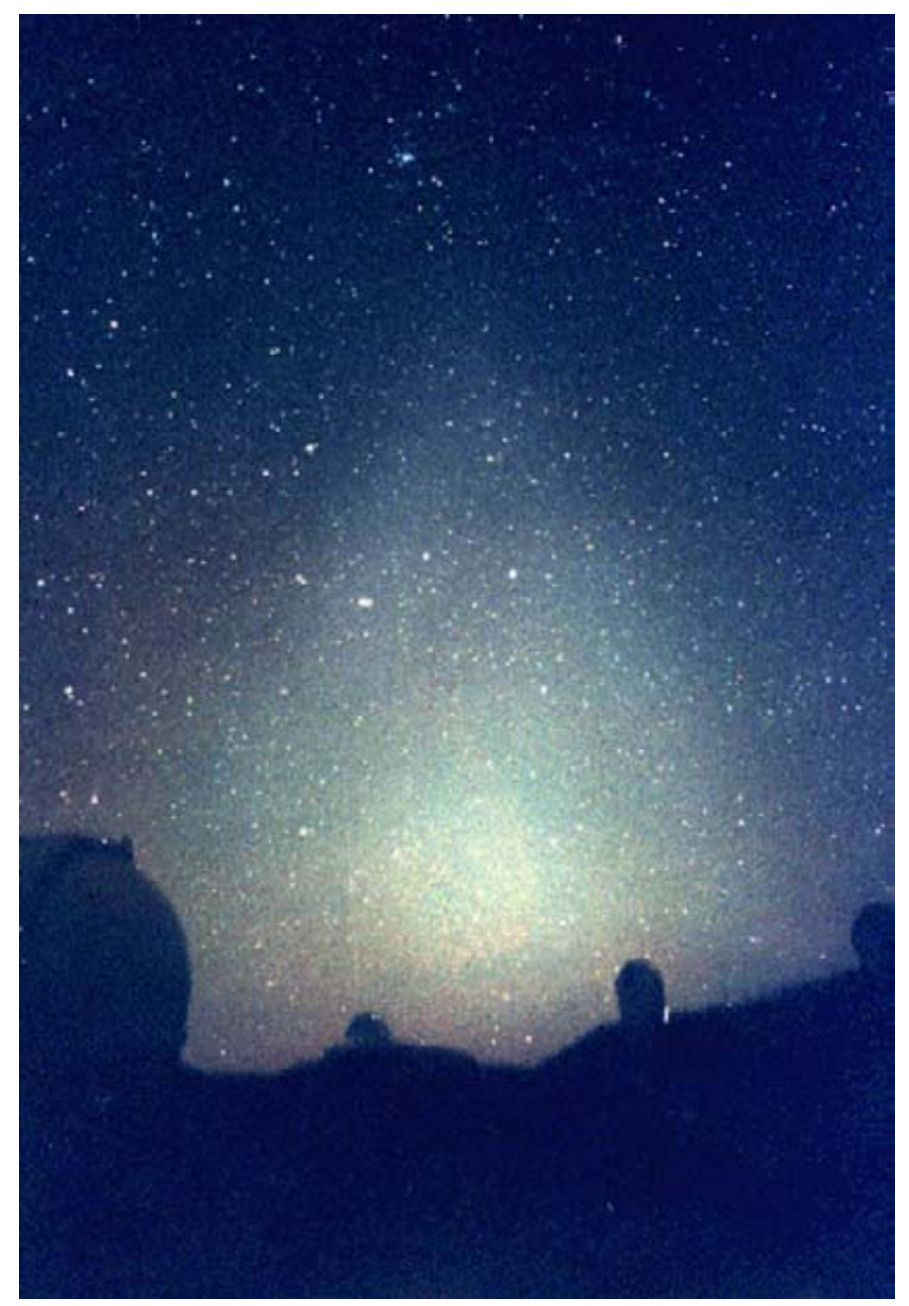

Figure 3. The Zodiacal light observed in the visible light from Manua Kea, Hawaii with an analog (35 mm-film) camera with wide angle lens (focal length $=28 \mathrm{~mm}$, Mukai and Ishiguru, personal communication). The brightness is produced by light scattering at dust of sizes 1 to $100 \mu \mathrm{m}$ distributed near $1 \mathrm{AU}$.

\subsection{Meteor Observations}

High velocity impacts of meteoroids cause the meteor phenomenon in planetary atmospheres (cf. Ceplecha, Borovi, Elford et al. (1998)): A meteoroid that enters the Earth atmosphere and atoms ablated from the meteoroid collide with atmospheric constituents. Meteoroids, atmospheric atoms and molecules undergo dissociation and ionization and form an expanding column of partially ionized plasma along the trajectory of the meteoroid. This generated plasma cloud in the atmosphere is commonly called meteor. The meteor trail is the extended radiation observed behind the meteoroid body. The meteor head is the plasma in the immediate surrounding of the meteoroid body that moves with the speed of the meteoroid.

The sizes of entering bodies for the different detection techniques range from about $10 \mu \mathrm{m}$ and beyond, depending on the mass, material composition, structure and 
entry speed. Some meteors are observed in meteor showers but the majority is observed as sporadic meteors. The detection of interstellar meteors with radar techniques was also reported (Taylor, Baggaley \& Steel 1996; Baggaley \& Neslusan 2002 and references therein; Meisel, Janches \& Mathews 2002). The classification of the meteors as interstellar is based on their derived heliocentric velocity. The orbital speeds derived from some of the radar techniques, however, are still subject to debate among researchers (Hajduk 2001), as are the selection effects of this technique. Head echo observations were recently used to derive the velocity vectors of in-falling particles, but the analyses are still limited by statistics (Meisel, Janches \& Mathews 2002; Janches, Pellinen-Wannberg, Wannberg et al. 2002). Head echoes are radar reflections that occur at the head when the produced ion cloud reaches a critical density (Pellinen-Wannberg \& Wannberg 1994).

Recent spectroscopic observations brought progress in understanding the composition of the entering bodies (see review by Borovicka, this issue). The observations indicate the diversity in the material composition of the meteoroid particles on scales of millimeters. Material properties for meteors on near-ecliptic orbits also differ from those of meteors in orbits with higher inclination. This possibly points to different properties of cometary and asteroidal meteoroids since cometary meteoroids can be expected to be more abundant at higher latitudes.

\subsection{Collected Samples}

While meteors are observed as a result of the heating and melting of the in-falling cosmic meteoroids, smaller particles are only moderately heated during entry into the atmosphere. The smaller particles are collected in the stratosphere (Brownlee 1985) or extracted from ice samples where concentration processes that occur in melt zones allow their collection in large quantities (Maurette, Hammer, Reeh et al. 1986). The exact size limits for particles to survive atmospheric entry are not clearly determined since survival depends on a variety of different parameters connected to the entry velocity and to the conditions of re-radiation of the entry heat. The size limits of the collected and analyzed particles are mainly determined by collection and handling methods. Cosmic dust particles collected in the stratosphere by high flying aircraft cover the size ranges from 5 to $50 \mu \mathrm{m}$; these particles are often denoted as interplanetary dust particles (IDPs) (Brownlee 1985). Cosmic dust particles that are collected from Antarctic ice and Greenland ice samples as well as from the ocean floor have typically sizes of $20 \mu \mathrm{m}$ to $1 \mathrm{~mm}$ (Maurette, Olinger, Michel-Levy et al. 1991; Kurat, G., Koeberl, C., Presper et al. 1994).

The mineralogical and morphological properties of these collected particles have been studied by laboratory analyses and the results have been recently reviewed by several authors in different scientific contexts (Rietmeijer 1999; Messenger 2000; Jessberger, Stephan, Rost et al. 2001; Rietmeijer 2002). Contamination from atmospheric constituents was detected in IDPs (see for instance Rietmeijer 1993) and some of the larger particles, extracted from ice samples are partially melted (Maurette, Olinger, MichelLevy et al. 1991; Kurat, G., Koeberl, C., Presper et al. 1994).

The presence of solar-wind noble gases (Hudson, Flynn, Fraundorf et al. 1981) confirms the extraterrestrial nature of the IDPs. Also nuclear tracks, in majority generated by solar energetic particles ('solar flare tracks'), have been identified in collected stratospheric cosmic dust (Bradley, Brownlee \& Fraundorf 1983). Exposure ages within the inner solar system derived from the tracks are approximately 10000 years (Bradley, Brownlee \& Fraundorf 1983). Compositional characteristics, such as isotope composition (Jessberger, Stephan, Rost, et al. 2001) ascertain the cosmic origin of the particles. Particles extracted from Antarctica ice samples showed elemental compositions similar to chondrites, and 
for a fraction of particles the extraterrestrial origin was confirmed by isotopic analysis of trapped neon (Maurette, Olinger, Michel-Levy et al. 1991).

The thermal history of the dust particles can be used to estimate their possible parent bodies. Flynn (1989) estimated from initial orbital parameters and entry processes the heating of the dust particles: The atmospheric-entry conditions inferred for the major fraction of the collected stratospheric cosmic dust is consistent with parent bodies in the main asteroid belt. Flynn (1989) further derived that cometary dust from parent bodies with perihelia greater than $1.2 \mathrm{AU}$ is heated in atmospheric entry to temperatures of approximately $900-1100 \mathrm{~K}$, and dust from comets with smaller perihelia is heated to temperatures beyond $1100 \mathrm{~K}$. The heating history does, however, not allow for a unambiguous determination of the parent body: Depending on the specific orbital history asteroidal dust may cross Earth orbit with a high velocity typical for dust released from a comet and vice versa. So cometary dust may have small velocities and therefore resemble from entry thermal processing an asteroidal dust particle. It is also quite possible that some collected stratospheric particles originate from the Kuiper belt. For the case of two specific particles, the density of solar flare tracks clearly exceeded the values that are typical for dust from comets or asteroids, which led Flynn (1996) to suggest they originate from the Kuiper belt. Also the capture of interstellar dust is possible, especially since they are focused by solar gravitational attraction (Mann \& Kimura 2000); their sizes, however, are typically below $5 \mu \mathrm{m}$, wich is currently the limit for the analyzed collected IDPs.

Results of the laboratory analyses provide evidence that some of the materials in the collected IDP samples are very pristine. The so-called chondritic IDPs are thought to be among the most primitive samples (Bradley 1994). For some of them there is evidence they originate from comets. By measuring the He release pattern upon laboratory heating Joswiak, Brownlee, Bradley et al. (1996) could infer the entry speeds of the thus analysed particles: Those particles for which they established cometary origin were in all cases porous chondritic IDPs with unique glass/metal compounds (i.e. GEMS = glass with embedded metal and sulfides). Among them the so-called cluster IDPs are thought to be cometary dust, since their enhanced $\mathrm{D} / \mathrm{H}$ ratio suggests a pristine nature (Messenger 2000). These cluster IDPs contain high abundances of GEMS. It is suggested that GEMS are either interstellar silicate dust particles or be the oldest known solar nebula solids (Bradley 1994). Studies of anhydrous chondritic porous IDPs of probable cometary origin also show much of the $\mathrm{C}$ to be in the form of fine grained amorphous carbon (Rietmeijer 2000; Wooden 2002), plus domains of aliphatic and aromatic carbon (Flynn, Keller, Feser et al. 2003; Keller, Messenger \& Flynn et al. 2002). Also GEMS in anhydrous chondritic porous IDPs contain nano-phase $\mathrm{C}, \mathrm{Fe}$, and FeS that make these $0.1 \mu \mathrm{m}$ substructures optically dark.

\subsection{In-situ Measurements in the Interplanetary Medium}

In-situ measurements of interplanetary dust carried out from Earth orbiting satellites detect particles of sizes typically below several $\mu \mathrm{m}$. In-situ measurements are well fitted by a combined model of the mass distribution of dust flux density near 1 AU (Grün, Zook, Fechtig et al. 1985). The model also fits to meteor observations and analysis of microcraters on lunar samples. In addition, measurements onboard Pioneer, Voyager, Ulysses and Galileo determined dust fluxes in interplanetary space beyond 1 AU. Inside Earth's orbit, Helios measurements were carried out between 0.3 and 1 AU (Grün, Pailer, Fechtig et al. 1980). All measurements were carried out close to the ecliptic plane with the exception of Ulysses, which moved into orbit almost perpendicular to the ecliptic after a Jupiter flyby. Most experiments measure the mass and impact speed of 


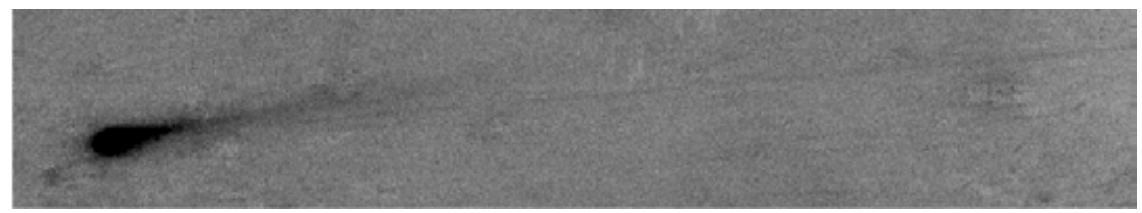

Figure 4. The dust coma and the faint dust trail of comet Churyumov-Gerasimenko taken by the Kiso $105 \mathrm{~cm}$ Schmidt telescope with R-band filters using a $2 \mathrm{~K}$ CCD camera (Ishiguro, Watanabe, Tanigawa et al. 2002). Observations of dust trails in the visible wavelength range allowed to derive the albedo of particles, which turned out to be very low.

particles. Deriving dust fluxes from the impact rates imposes some uncertainty, since derived numbers depend on assumptions for the orbital distributions of particles. Helios measurements indicate the presence of two distinct dust components: the major component is in low to medium eccentricity orbits near the ecliptic and a second component of dust is in orbit with presumably higher eccentricities and consists of dust of lower material strength (Fechtig 1982). The second component was interpreted to be made of cometary dust (Fechtig 1982).

The in-situ detection of interstellar dust entering the solar system is important for further understanding of the dust evolution. It was first identified in dust fluxes onto Earth orbiting satellite where the gravitational focusing of the interstellar dust particles caused a flux variation along Earth's orbit (Bertaux \& Blamont 1976). Clear identification of interstellar dust in the solar system was obtained from measurements aboard Ulysses since impacts speeds and directions are different from those of interplanetary dust (Grün, Gustafson, Mann et al. 1994). Ulysses measurements allow a comparison to astrophysical models of dust properties in the local interstellar medium (Landgraf 2000; Frisch, Dorschner, Geiss et al. 1999; Mann \& Kimura 2000; Mann \& Kimura 2001). The measured mass distribution of the interstellar dust in the solar system is influenced by different forces from which particle properties can be inferred (cf. Mann 1996). Namely, the repulsion by radiation pressure force changes the mass distribution of the measured interstellar dust with distance from the Sun along the Ulysses orbit (Landgraf 2000; Mann \& Kimura 2000; Mann \& Kimura 2001). Kimura, Mann \& Jessberger (2003) showed that the radiation pressure force infered from the data agrees with a model of core-mantle dust particles that form larger agglomerates. The core-mantle dust particles were simulated with sizes of $100 \mathrm{~nm}$, while bare silicates and bare carbonaceous materials may be present as dust particles smaller than $10^{-14} \mathrm{~g}$.

\subsection{Observations of Cometary Dust}

Comets are visible when they release gas and dust during that parts of their orbits which are sufficiently close to the Sun such that melting of volatiles in the nucleus causes this activity. Dust in the vicinity of comets is observed in their comae and tails and as a result of the formation history of comets the dust is expected to be more pristine than dust from other parent bodies (see Figure 5). Observations over a wide range of wavelengths allow today studying the gas and dust surrounding the cometary nucleus. A discussion of the dust and gas observations will be given in the following section 3 .

Refined observations allow to detect the faint brightness of the larger dust particles ejected from comets (see Figure 4). Narrow trails of dust coincident with the orbits of periodic comets have been discovered in the data of the Infrared Astronomical Satellite (IRAS) (Sykes, Lebofsky, Hunten et al. 1986) and are detected with the Infrared Satellite Observatory (ISO) and with Spitzer Space Observatory. Within the IRAS data trails were studied in detail for 8 comets; alltogether more than 100 faint dust trails are suggested 


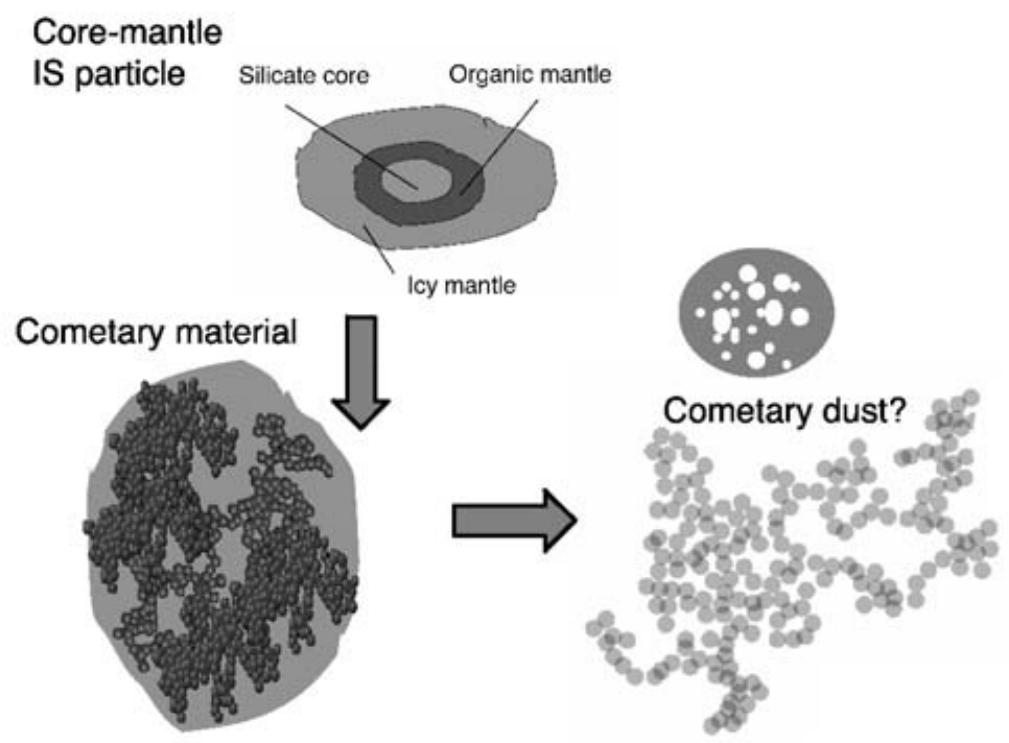

Figure 5. Formation of cometary matter: a plausiblescenario of the formation of cometary matter is that interstellarcore-mantle particles are (partially altered and) incorporated intothe cometary nucleus. The icy materialforms the matrix of the cometary matter while silicate coresand organic refractory mantles may partially survive and provide thebasic constituents of the cometary dust observed in the solar system.

by the IRAS data (Sykes \& Russell 1992). The particles are in orbits close to that of the parent comet and seen both ahead and behind the comet. Some trails exist even without an observable associated parent comet. Recently, Ishiguro, Watanabe, Tanigawa et al. (2002) have found a visible dust trail along the orbit of comet $22 \mathrm{P} / \mathrm{Kopff}$ and a survey (see Mukai, Ishiguro \& Usui 2002) showed that a large fraction of comets are also associated with visible trails. The trails consist of large (a few $\mathrm{cm}$ ) and dark (albedo of 0.01 ) dust particles (see Figure 4). As Mukai, Ishiguro \& Usui (2002) pointed out, the existence of dust trails along the orbit of parent comets seems to be general feature: Observations of comet trails stimulate re-evaluation of several cometary phenomena such as (1) the gas-dust interaction when the dust particles are ejected from the cometary nuclei, $(2)$ the mass-loss rate and aging of comets, and (3) the mass distribution of small solid bodies in the solar system.

Direct in-situ detection of cometary dust, though limited by experimental conditions, was possible with the space missions to comet Halley in the 1980's (Jessberger, Christoforidis \& Kissel 1988; Kissel, Brownlee, Büchler et al. 1986; Kissel, Sagdeev, Bertaux et al. 1986; Schulz, Kissel, \& Jessberger 1997). Data were acquired by Giotto to a minimum flyby distance of $600 \mathrm{~km}$, by the Vega spacecraft to a minimum distance of $8000 \mathrm{~km}$.

Recent measurements of dust fluxes were made during the encounter of DS1 at comet Borrelli (Tsurutani 2004) and during the Stardust flyby at comet Wild2 (Tuzzolino, Economou, Thanasis et al. 2004). The latter Stardust measurements show strong evidence of dust fragmentation taking place in the coma, as will be discussed below. 


\section{Cometary Dust Evolution in the Coma}

Dust is steadily lifted from the surface of the nucleus by the out-flowing gas or released when sublimation of ices below the surface of the nucleus breaks up the crust that is formed on the surface. The velocity of dust is initially determined by the flow of the surrounding gas, and at larger distances from the nucleus, by solar gravity and radiation pressure force. Local variations of the surface activity of the nucleus, chemical reactions in the gas phase as well as interactions between the gas and the dust phase influence the distribution and composition of coma gas. Extended sources of gas are the sublimation of ices and semi-refractory components in the dust, and chemical reactions in the gas phase. The highly refractory component of cometary organics remains within the dust particles that feed the solar system dust cloud.

\subsection{Dust and Coma Gas Observations}

\section{Observations and in-situ detection of coma gases}

Radio and sub-millimeter spectroscopic remote observations of comets show numerous lines from molecules, radicals and ions including the major carbohydrates as well as complex organic molecules (see Crovisier, this issue). The majority is directly produced by the outgassing of the nucleus. The presence of an extended source of coma gas is clearly seen in neutral gas measurements carried out on Giotto at comet Halley. When comparing the measured $\mathrm{H}_{2} \mathrm{O}$ and $\mathrm{CO}$ radial density profiles after correction for gas kinematics and geometry effects, they show completely different behaviors: The $\mathrm{H}_{2} \mathrm{O}$ profile outward from approximately $5000 \mathrm{~km}$ from the nucleus follows the radial decrease expected from the high photo destruction rate of the molecule in the solar UV radiation. The CO profile, in contrast, increases with distance from the nucleus out to $25000 \mathrm{~km}$. Further out the $\mathrm{CO}$ profile follows a flat slope expected from the low rate of photo destruction of $\mathrm{CO}$ compared to the flowing time in the coma. The CO profile between the nucleus and a distance of $25000 \mathrm{~km}$ is explained with an extended source being present in the coma (Eberhardt 1999). Remote observations of the spatial distributions of different species in many cases show evidence for an extended source. The coma composition also changes with heliocentric distance of the comets. Disanti, Mumma, dello Russo et al. (1999) derive from observations of Hale-Bopp that the extended CO source may only be effective for small heliocentric distances, while at larger distances the coma gas is predominantly produced by the nucleus.

\section{Observations of changing dust properties}

Observational data suggest that also dust properties and size are changing within the coma (see Figure 6). The generally elongated shapes of isophotes seen in ground-based observations, for instance, can be explained if particle sizes change with distance from the nucleus (Combi 1994). From analyzing comae radial brightness profiles, Baum, Kreidl \& Schleicher (1992) concluded that for 10 out of 14 comets the brightness decrease is steeper than would be expected from dust motion influenced by radiation pressure, meaning that either the albedo or the size of particles changes with distance from the nucleus.

Further results were obtained by recent high spatial resolution observations of brightness, polarization and colour of comet $2 \mathrm{P} /$ Encke. The polarization of the coma was observed to increase with projected distance from the nucleus, suggesting a change in the mean scattering properties of the dust particles on a time-of-flight timescale of 1 hr (Jewitt 2004). Jewitt (2004) suggests that disaggregation of composite, porous dust particles is a likely cause of the fragmentation. Subsequent observations confirm this result and interpretation (Jockers, Kiselev, Bonev et al. 2005). 


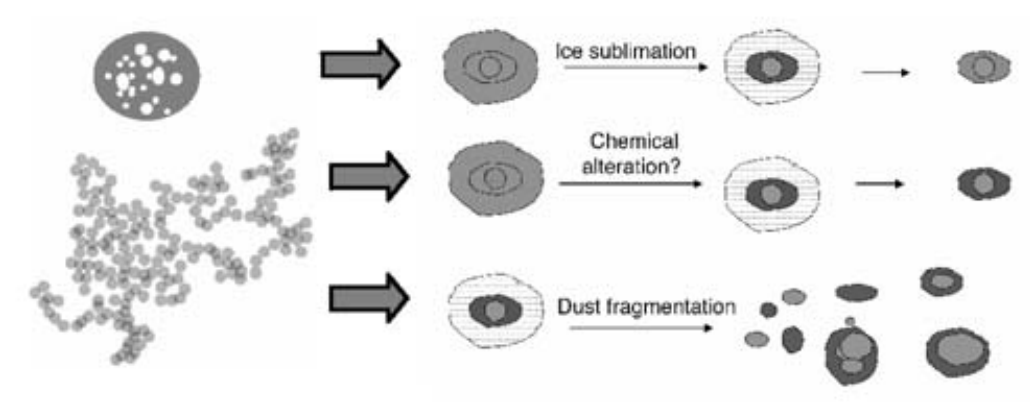

Figure 6. A possible path of dust processing in the coma: the pristine cometary material is altered by fragmentation, sublimation and chemical reactions in an unknown way. This illustration follows the Greenberg model describing the cometary dust as agglomerates of core-mantle particles.

In addition to changes in dust particle sizes and dust composition, variations of the activity over the surface of the nucleus influence the observed coma profiles. Imaging the inner coma of comet $\mathrm{P} /$ Halley over a $10^{4} \mathrm{~km}$ square region showed that the near-infrared colors between 1 to $5 \mu \mathrm{m}$ were not constant as a function of nucleocentric distance and moreover the radial decrease in surface brightness significantly differed from the jet-side of the nucleus to the opposite, tail side (Woodward, Shure, Forrest et al. 1996). Observations of the coma of comet Tabur prior to the perihelion passage are also interpreted with either the existence of different particle population or with dust destruction: The scattered continuum light from dust detected with visible spectroscopic observations was blue within $5300 \mathrm{~km}$ around the projected position of the photocenter and reddish further Sunward Turner \& Smith (1999). Broadband (B, V, R) CCD imaging of the comet proves that the reflectance of the dust behaves differently in the Sun and in the tail directions (Lara, Schulz, Stüwe et al. 2001).

Aside from the change of dust properties within the coma, properties vary with the heliocentric distance. Narrowband filter photometry observations of comet Hyakutake indicate a change in dust color from significantly reddened at large heliocentric distances to close to the solar spectrum at small heliocentric distance (Schleicher \& Osip 2002). The authors suggest the change of color with heliocentric distance implies a significant change in dust particle sizes or a changing proportion of different dust populations. For comet Hale-Bopp the observed 3 to $20 \mu \mathrm{m}$ spectral energy distributions derived from observations were used to fit thermal emission models (Harker, Wooden, Woodward et al. 2002, 2004): Near perihelion, Hale-Bopp displayed higher jet activity than at larger heliocentric distance and the coma dust had a steeper size distribution, was more porous and had more crystalline silicates. The authors suggest, that these changes indicate either the fragmentation of aggregate dust particles, or that the dust particles released from highly active areas were different, possibly more pristine.

Variation of the properties from comet to comet is possibly more pronounced than this variations with heliocentric distance. Baum, Kreidl \& Schleicher (1992) infer from their study that large intrinsic differences exist in the nature of the dust particle populations 
of the 14 considered comets, while they could not find a systematic connection of the porperties to the heliocentric distances of the comets.

\section{In-situ dust flux measurements}

The Dust Flux Monitor Instrument (DFMI) aboard Stardust measures particles in the $10^{-14}$ to $10^{-7} \mathrm{~kg}$ mass range. During the flyby at Comet $81 \mathrm{P} /$ Wild 2 it encountered regions of intense swarms of particles. The clouds of particles were only a few hundred meters across, which is explained by particle fragmentation (Tuzzolino, Economou, Thanasis et al. 2004; Green, McDonnell, McBride et al. 2004). Imaging also reveals a large numbers of jets projected nearly around the entire nucleus (Tsou, Brownlee, Anderson et al. 2004). Sekanina, Brownlee, Economou et al. (2004) interpret the dust flux measurements with the release of sheets of high dust density extending from small sources on the rotating nucleus. They suggest that the dust is accelerated by the expanding gas in the jets and that large cometary fragments may travel with the comet for a long time and then possibly fragment as a result of sublimation. Clark, Green, Economou et al. (2004) suggest that fragmentation causes these streams and that fragmentation itself could be caused by enhanced heating as well as depressurization, phase transitions, exothermic chemical reactions, centrifugal forces, and electrostatic repulsion. Green et al. (presented at IAU Symposium No. 229) pointed out that the mass distribution measured with DFMI changed significantly during the encounter, which they also interpret as due to fragmentation processes.

\section{In-situ dust composition measurements}

In-situ mass spectrometer measurements were made as close as $600 \mathrm{~km}$ from the nucleus of comet Halley. The masses and densities of the dust particles measured with the mass spectrometers aboard the Giotto and Vega spacecraft range from $10^{-19}$ to $10^{-14} \mathrm{~kg}$ (approximate diameter range 0.02 to $2 \mu \mathrm{m}$ ) with densities from 0.3 to $3 \mathrm{~kg} / \mathrm{m}^{3}$. The interpretation in terms of the elemental compositions of the impacting dust particles requires a detailed understanding of the impact ionization process. Though the understanding of the impact ionization process is limited, the published results (Jessberger, Christoforidis \& Kissel 1988; Kissel, Brownlee, Büchler et al. 1986; Kissel, Sagdeev, Bertaux et al. 1986) provide interesting information about the dust in the coma. The detected particles are mixtures of two end-member components, often called CHON (rich in the elements H, $\mathrm{C}, \mathrm{N}$, and $\mathrm{O}$ ) and ROCK (rich in rock-forming elements as $\mathrm{Si}, \mathrm{Mg}, \mathrm{Fe}$ ). The $\mathrm{CHON}$ component is assumed to be refractory organic material while the ROCK component is assumed to consist of silicates, metals, and oxides. Further analysis by Schulz, Kissel \& Jessberger (1997) showed the ROCK dominated particles are composed of primarily Mg-rich pyroxenes, some Mg-Fe pyroxenes and olivines and relatively rare Fe and FeS; about $70 \%$ in Fe and FeS particles.

The silicate and the carbonaceous component are mixed down to the finest scale, possibly (but not necessarily) in the form of a core-mantle structure. CHON- and ROCKdominated particles make up each about $25 \%$ of the measured dust and most of the small particles (below $10^{-17} \mathrm{~kg}$ ) are rich in the light elements $\mathrm{H}, \mathrm{C}, \mathrm{N}$, and O. Lawler \& Brownlee (1992) found from their analysis of the mass spectra that there are essentially no pure $\mathrm{CHON}$ particles in the $0.1-1$ micron size range. Given the uncertainties of the measurement, the presence of pure $\mathrm{CHON}$ particles of even smaller sizes can not be unambiguously concluded from the data. The fact that the ${ }^{12} \mathrm{C} /{ }^{13} \mathrm{C}$ isotope ratios vary from particle to particle is interpreted that there has been no process leading to chemical homogeneity in the history of cometary dust (Jessberger, Christoforidis \& Kissel 1988). Fomenkova \& Chang (1997) point out that that some detected carbon-rich particles have ${ }^{12} \mathrm{C} /{ }^{13} \mathrm{C}$ isotope ratios similar to AGB stars. 


\subsection{Models of Gas and Dust Interactions in the Coma}

Understanding the coma processes is essential for interpretation of observational data, but the complexity of coma phenomena does not allow a simple model to describe all the processes at the same time. Dust is steadily lifted from the surface of the nucleus by the out-flowing gas but also strongly variable dust and gas production occurs. The amount of ejected dust varies. Hale-Bopp produced, for instance, gas jets that were not associated with observable dust signatures (Lederer, Osip, Thomas-Osip et al. 2005). Solar radiation pressure force and gas drag force acting on the dust, together with dust structure and composition determine the dynamical evolution of dust. The size dependences of the acting forces induce relative velocities of more than $0.5 \mathrm{~km} / \mathrm{s}$ within the dust component, as do the complex motions of coma gas (Combi, Kabin, DeZeeuw et al. 1997).

Describing the out-gassing of the nucleus and the evolution of its circum-nuclear coma with a steady-state outflow of gas and dust is already numerically complex (Crifo \& Rodionov 1999). To describe the extended source of gas due to ice sublimation Crifo (1995) has developed a numerical two-step model, which accounts for surface ejection of fragments of water ice mixed with mineral dust particles, that subsequently sublimate in the coma. The calculations achieve a better agreement with the measured $\mathrm{H}_{2} \mathrm{O}$ velocity profiles in comet Halley than steady-state outflow models. Verifying models of dust-gas flows is difficult, since these are not the only parameters to determine dust and gas in the coma. Konno, Hübner \& Boice (1993) study, for instance, dust fragmentation in near-nucleus jet-like features at comet Halley and come to the conclusion that dust fragmentation alone does not explain the dust observations.

To achieve a better description of the coma, Hübner \& Benkhoff (1999) consider three sources for coma gas: (1) release of water vapor from the surface of the nucleus, (2) release of other, more volatile, species from the porous interior of the nucleus, and (3) a distributed source releasing gases from ices and volatiles contained in the dust. Greenberg \& $\mathrm{Li}(1998)$ point out that the distribution of $\mathrm{CO}, \mathrm{C}_{2}, \mathrm{C}_{3}, \mathrm{CN}, \mathrm{H}_{2} \mathrm{CO}$ can not be explained as daughter molecules originating from more complex gas phase species. They apply a highly porous silicate core organic refractory mantle model to explain the presence of these coma species and the amount of CO in Comet Halley. By computing the heating of fluffy aggregates of interstellar core-mantle particles they estimate a maximum $\mathrm{CO}$ production rate that is still significantly less than the values needed to explain the observed CO. Differences between the model and the observations can arise from various factors such as the assumed dust to gas ratio, the dust fragmentation and sublimation processes, or overestimation of the extended $\mathrm{CO}$ abundance.

The different carriers of extended gas sources are currently not quantified. The fragmentation and vaporization of dust is commonly discussed as one of the possible sources of spatially extended coma gases (see cf. Festou 1999). Organic dust components in particular, are suggested to explain observations (e.g. Bockelee-Morvan \& Crovisier 2002; Disanti, Mumma \& Dello Russo et al. 2001). Chemical reactions within the gas phase are also a possible source: Bockelee-Morvan, Crovisier, Mumma et al. (2005) explain the increase in $\mathrm{CO}$ in comet Hale-Bopp near perihelion (between solar distances of $0.93 \mathrm{AU}$ and $1.5 \mathrm{AU}$ ) as a consequence of $\mathrm{CO}$ excitation mechanisms. Aside from the other uncertainties outlined in this section, uncertainties of the dust models arise from a lack of knowledge about the chemical appearance of the organic refractory material. It is therefore difficult to estimate the optical properties and the sublimation rates of the cometary dust. 


\section{Cometary Dust Optical Properties}

The optical properties of cometary dust derived from observations are known for many comets and therefore provide a good basis for analysis. Differences in the optical properties of cometary dust can come from particles processing in the coma, particle fragmentation in the coma, as well as differences of material from comet to comet. Nevertheless, visible light observations indicate common characteristics in the albedo, polarization and colour of the particles, which differ from other cosmic dust populations. They agree with models of porous dust consisting of silicates and absorbing, possibly organic refractory materials. Silicate features, appear in several comets and indicate the existence of both, amorpous and crystalline silicates. The high amount of absorbing material and its close mixture to the silicate, might be the special characteristic of the cometary dust.

\subsection{Albedo and Polarization Observations}

Optical and near IR observations allowed to study the albedo and linear polarization of cometary dust (Dobrovolsky, Kiselev \& Chernova 1986; Dollfus, Bastien, Le Borgne et al. 1988; Kolokolova, Hanner, Levasseur-Regourd et al. 2004, Kelley, Woodward, Jones et al. 2004). Improved observation techniques and the recent apparitions of the bright comets Hale-Bopp and Hyakutake have further increased the amount of optical data (Hadamcik \& Levasseur-Regourd 2003; Kiselev \& Velichko 1998).

Regardless of the differences in the properties of comets, the dust particles have common characteristics in their optical properties:

- The albedo is low compared with other atmosphere-less bodies in the solar system. The albedo gradually increases with wavelength from the optical to the near-infrared wavelength range.

- The brightness smoothly changes with phase angle and shows strong and weak enhancements toward large and small phase angles, respectively.

- The linear polarization in relation to phase angle is described as a bell-shaped curve with a broad maximum around a phase angle of $90^{\circ}$ and a shallow negative branch of the polarization at small phase angles.

- The polarization increases with wavelength, while in the negative branch at small phase angles it is constant or decreases with wavelength.

Several studies have been carried out to fit the observed data for albedo and linear polarization in the visible wavelength range. In many cases light scattering and absorption properties of cometary dust were simulated based on Mie theory that provides rigorous solutions for interaction between electromagnetic radiation and homogeneous spheres (Mukai \& Koike 1990; Mukai, Mukai \& Kikuchi 1987). Developments of light-scattering theories combined with increased computer capabilities allowed to assume more realistic morphologies (Lumme \& Rahola 1994; Petrova, Jockers \& Kiselev 2000; Draine 1988; Draine \& Flatau 1994).

A model to describe cometary dust as aggregates of submicron monomers achieved, for the first time qualitative simultaneous agreement with the four observed optical characteristics listed above (Kimura, Kolokolova \& Mann 2003; Kolokolova, Kimura \& Mann 2004; Mann, Kimura \& Kolokolova 2004). The size of the constituent monomers in the model is $100 \mathrm{~nm}$, which is in accord with the average size of constituent dust particles of IDPs (Brownlee 1978; Jessberger, Stephan, Rost et al. 2001). The obtained results are similar for the considered two types of irregular dust structures. The importance of the assumed scattering properties lies in the assumption of the monomer size and of the refractive index. An increase of both $n$ and $k$, where $n$ and $k$ are the components of the optical constant $m=n+i k$, is most suitable for obtaining the optical properties 

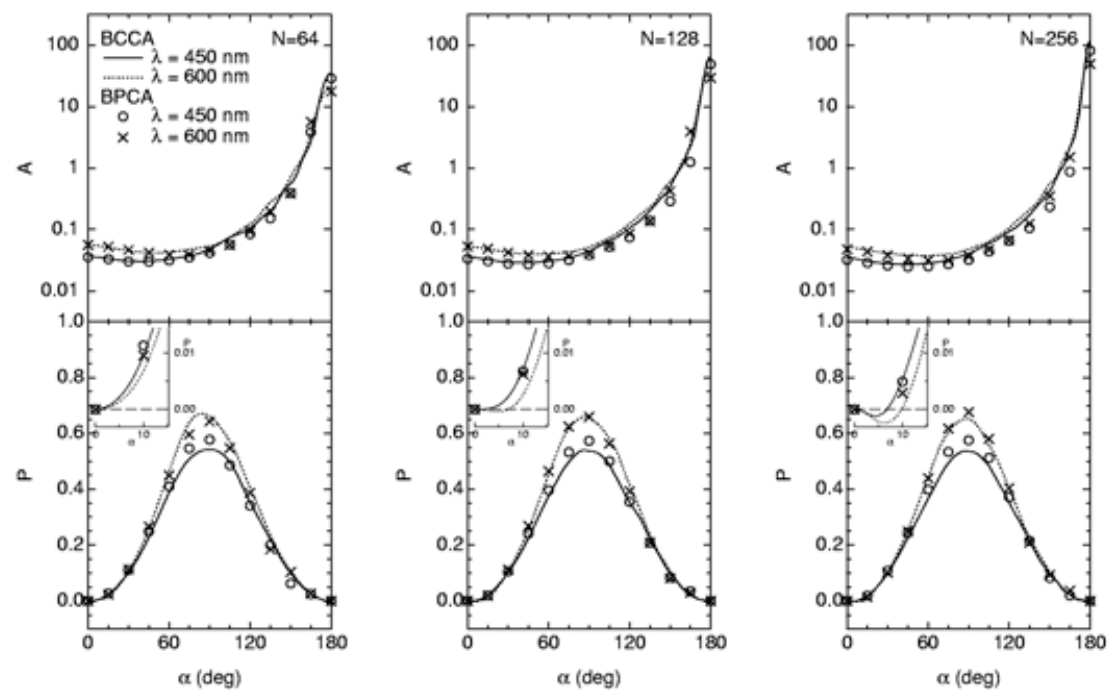

Figure 7. Optical properties of dust: Shown are the geometric albedo $A$ and polarization $P$ calculated for two different models of fluffyaggregate particles. The particles are agglomerates of identical homogeneous spheres. The numbers of monomers are $N=64,128,256$. BPCA particles (symbols) and BCCA (lines) particles denote different structures of the aggregates. The calculations were made at wavelength of 450 and $600 \mathrm{~nm}$, see Mann, Kimura \& Kolokolova (2004).

(Mann, Kimura \& Kolokolova 2004). Previous studies have shown already, that particles consisting of a silicate core and an organic refractory mantle material can produce a characteristic silicate emission feature (Greenberg \& Hage 1990). This model, developed to explain albedo and polarization, may therefore also reproduce thermal properties of cometary dust.

For the calculated cases of particle sizes (see Figure 7), limited by the computer capacities, the obtained maximum polarization is higher than the observed values and the negative polarizaton is small. The authors expect a better agreement for larger particle sizes as well as possibly for aggregates of non-spherical particles. Further studies of this dust model should include a comparison with thermal observations as well as a systematic study of the influence of the dust structure on the results (Mann, Kimura \& Kolokolova 2004). Also dedicated laboratory measurements are currently developed (Hadamcik, Renard, Levasseur-Regourd et al. 2003).

\subsection{Thermal Emission Data}

Thermal emission in the infrared wavelength range gives an insight into the mineral composition of cometary dust (Becklin \& Westphal 1966; Hanner \& Bradley 2005). Observing the mineralogy of cometary silicates has interesting implications for the formation of the comets: Cometary silicates are most likely a mixture of (1) remnants of interstellar silicates which are amorphous and (2) crystalline silicates. The crystalline silicates have two possible origins: (a) solar nebula condensates or (b) amorphous (interstellar silicate) annealed in the solar nebula. Crystalline silicates are formed in the solar nebula if cooling is slow enough. On the other hand, if the cooling is fast, amorphous silicate will condense even in the solar nebula.

Characteristic for silicate particles are emission features in the $10 \mu \mathrm{m}$ and in the 16 $35 \mu \mathrm{m}$ spectral regime. The structure and intensity of the emission features depends on the particle size, the structure and even the temperature of particles and therefore the 
interpretation of the observed spectra is difficult. Spectroscopic observations show evidence for certain silicate minerals in the infrared spectra (Campins \& Ryan 1989; Hanner, Gehrz, Harker et al. 1997; Hanner 1999; Hanner, Lynch \& Russell 1994). According to observations, silicates in comets appear to comprise both crystalline Mg-rich silicates and Mg-Fe glassy or amorphous silicates (Wooden, Harker, Woodward et al. 1999). Strong features have been observed for the majority of Oort cloud comets while weak emission features have been observed for only a few short period comets. Hanner (2003) points out that this absence is either due to a different composition or due to a lower abundance of submicron sized dust particles in the short period comets. Kelley, Woodward, Jones et al. (2004) point out that from their polarimetry and near-infrared photometry for six comets the high-polarization comets are characterized by moderate to strong midinfrared silicate emission, while the low-polarization comets have a weak silicate emission feature or the feature is absent. The mineralogy of cometary dust underlying the different observational results was also discussed by Wooden, Harker \& Woodward (2000); Wooden (2002); Harker, Wooden, Woodward et al. 2002, 2004) Hanner (2003), Hanner \& Bradley (2005), and Wooden, Charnley \& Ehrenfreund (2005). Valuable information about the properties of pristine dust ejected from the interior of the nucleus are expected from observation campaigns connected to the Deep Impact mission (see A'Hearn, this issue).

The mineral identifications from the 10 and $20 \mu \mathrm{m}$ cometary spectra are consistent with the composition of anhydrous chondritic porous aggregate IDPs (Hanner 1999; Bradley 1988; Bradley, Keller, Snow et al. 1999; Wooden 2002). This again support the hypothesis that the latter are of cometary origin.

\subsection{Classification of Comets by Optical Properties}

In spite of the similarities of the optical properties when comparing the cometary dust to other dust particles in the solar system, the cometary dust shows a broad range of different properties. Dobrovolsky, Kiselev \& Chernova (1986) and subsequently LevasseurRegourd, Hadamcik \& Renard 1996, suggest the existence of two classes of comets for which the optical, linear polarization vs. phase angle relations fall into two distinct groups: best-distinguished at large phase angles, the high polarization comets (including West 1976 VI, P/Halley 1986 III and Levy 1990 XX) have maximum polarizations $P_{\text {max }} \approx 30 \%$ while the low polarization comets (e.g. Kobayashi-Berger-Milon 1975 IX, Austin $1990 \mathrm{~V}$ ) have $P_{\max } \approx 20 \%$. The recent numerous observations of comet Hale-Bopp, however, do not clearly fit within these two groups Kiselev \& Velichko 1999; Manset \& Bastien 2000; Hadamcik \& Levasseur-Regourd (2003). Moreover, the two classes of comets that Levasseur-Regourd, Hadamcik \& Renard (1996) suggest show no clear relationship to the dynamical properties of the comets. It is therefore doubtful whether the comets can be clearly distinguished into two different groups of different dust properties.

We expect that variation of particle properties within comets to significantly influences observational results. Observations obtained before the 1990's have not been made with imaging detectors (Kiselev \& Velichko 1999) and therefore interpretation might be difficult. Gas pollution of early polarization data may hamper the results, as indicated, for instance by recent observations of Comet Encke (Jewitt 2004; Jockers, Kiselev, Bonev et al. 2005). There is the possibility that the variation of albedo and colour occurs within the comet, as was seen with spatially resolved observations.

The recent, spatially resolved observations, however, show radial gradients and localized structures in the polarization data (Kolokolova, Jockers, Gustafson, \& Lichtenberg 2001; Jockers, Rosenbush, Bonev et al. 1999) as well as higher polarization in the region of coma jets (Jockers, Rosenbush, Bonev et al. 1999; Furusho, Suzuki, Yamamoto et al. 
1999; Hasegawa, Ichikawa, Abe et al. 1999). Polarization measurements may therefore highlight the difference in properties of dust released by discrete active areas. Jets may release dust from the nuclear subsurface that has not been subjected to weathering on the parent body. If so, polarization measurements may provide additional about different dust components within a comet.

\subsection{Current Models to Describe Optical Properties}

The recent model to describe albedo and polarization of cometary dust contradicts the long-lasting paradigm that cometary dust is dark because it is fluffy. The new model calculations show that absorbing material - possibly organic refractory - is closely connected to silicate into the smallest scales. This might explain the common characteristics of cometary dust compared to other dust species. The model of core-mantle particles as the sub-structure of the cometary dust is intriguing since it provides a direct connection to the interstellar core-mantle particles as they possibly exist in molecular clouds. The molecular cloud material, however is processed during the solar system formation and subsequent evolution. Therefore this picture of the cometary dust as agglomerate of interstellar particles is too simple. It is quite plausible that aggregates build out of monomers with different material composition provide a more realisitc description of the cometary dust. On the other hand, the simple model may be adequate for describing the average optical properties: Since the carbonaceous monomers are relatively dark, we can expect them to determine the scattering properties of an aggregate even when other types of monomers exist.

It still has to be investigated, how properties of cometary dust in the Zodiacal cloud would appear. We expect, that the particle processing in the interplanetary medium, though possibly present, will cause comparably small changes. Reach, Morris, Boulanger et al. (2003) studied the Zodiacal emission with ISOCAM observations on ISO. They report excess emission of $6 \%$ of the continuum in the $9-11 \mu \mathrm{m}$ range, which could be matched by a mixture of Mg-rich amorphous silicate, dirty crystalline olivine and a hydrous silicate (montmorillonite). In the data that range from solar elongations $68^{\circ}$ to $113^{\circ}$ and from the ecliptic plane to the pole, they note a tendency that the strength of the features increases toward the Sun and toward high latitude above the ecliptic. This possibly indicates that the Zodiacal light is more influenced by cometary dust at high latitudes above the ecliptic and at small distances from the Sun. The difficulties of the line-of-sight inversion, however, do not allow to clearly follow the cometary dust properties in the interplanetary medium.

\section{Constituents of the Solar System Dust Cloud}

The relative contributions of different sources to the overall solar system dust cloud change within the solar systems as well as they depend on the considered measurement technique. Certainly dust from asteroids provides a large component of the dust near Earth orbit, but it is also preferably seen with most of the detection methods. Dust from comets becomes increasingly important at small distances from the Sun inward from 1 AU where collisions of cometary meteoroids provide the majority of dust production. Destruction of cometary dust and meteoroids including the carbon-bearing species feeds the pick-up ion component of the solar wind.

\subsection{Dust Dynamics}

The major effects that determine the distribution of dust in interplanetary space are solar gravitation, solar radiation pressure, mutual catastrophic collisions, and the influence of 
the Lorentz force on electrically charged dust particles. After being released dust particles stay initially in orbits similar to (but not identical to) those of their parent bodies. The influence of the planets inward from Jupiters orbit, aside from local effects, causes orbital perturbations as seen in the symmetry plane of the dust cloud. Orbital resonances at the outer planets and subsequent ejection in hyperbolic orbits are common for dust particles originating from the Kuiper belt and limit the amount of Kuiper belt dust that reaches the inner solar system (Liou, Zook, Dermott 1996). The particles that stay in the solar system are decelerated by the Poynting Robertson (P-R) effect and the Plasma PoyntingRobertson effect and therefore approach the Sun. Typical timescales of the P-R lifetime at $1 \mathrm{AU}$ range up to $10^{5}$ years, depending on the mass and scattering properties, which determine the radiation pressure force. Particles are formed and destroyed by mutual catastrophic collisions. Collisions limit the lifetime of large particles and provide a source of smaller particles, with the dividing mass between the smaller and the larger particles at approximately $10^{-10}$ to $10^{-9} \mathrm{~kg}$.

As a result the particles seen in the Zodiacal light form an approximately rotationally symmetric dust cloud. All the inclination distributions agree with a concentration of the dust cloud toward the ecliptic plane. Namely, dust from asteroids and short-period comets produce the concentration of the dust cloud in the ecliptic plane. The distributions of inclinations derived from different Zodiacal cloud models peak more strongly at small inclinations than does the distribution of the inclinations in the orbits of the sporadic meteors (Kneissel \& Mann 1991). Model calculations indicate that mutual collisions of dust inward from $1 \mathrm{AU}$ could shift the size distribution to smaller particles (Ishimoto \& Mann 1999; Ishimoto 2000). Since observational data show no evidence of this, the lost particles need to be replenished.

\subsection{Different Sources}

The overall picture of a homogenous dust cloud of particles produced in the outer solar system and drifting toward the Sun as a result of the P-R effect is not fully correct and does only apply to the region near Earth orbit. Composition, structure and size distribution of dust change with latitude and with distance from the Sun within the dust cloud, as does the relative contribution of the different sources. The relative amount of dust from the different sources within the dust cloud is uncertain and varies spatially within the dust cloud and possibly also with time. Also the detection methods are biased and therefore different types of observations indicate the preponderance of different types of particles. The picture of the solar system dust cloud seems to be as follows: The majority of dust outward from 5 AU originates from the Kuiper belt and the local interstellar medium. The dust cloud near $1 \mathrm{AU}$ contains to a large extent dust from comets and asteroids, with most observations, i.e. analysis of collected samples, Zodiacal light analysis, indicating the preponderance of asteroidal dust. While observations of the Zodiacal light indicate the stability of the overall dust cloud near 1 AU over scales of years and decades, this is not the case for the inner solar system dust cloud where observational data are limited. Model calculations of the collision evolution indicate that the dust inward from 1 AU needs to be locally replenished. The collisional fragmentation of cometary meteoroids is the most plausible source. It is also possible that local dust production from cometary meteoroids inside 1 AU leads to changes of the dust cloud composition on time scales of years (Mann, Kimura, Biesecker et al. 2004). This would explain differences in the F-corona brightness observed during eclipses in different years (Kimura, Mann \& Mukai 1998; Ohgaito et al. 2002). 


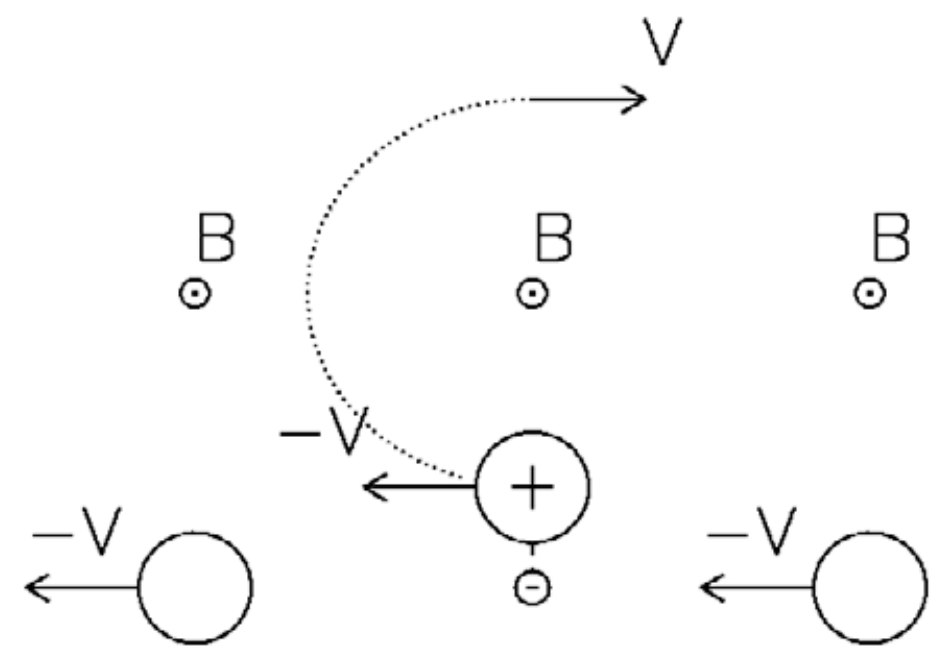

Figure 8. A schematic view of the pick-up process in the solar wind plasma frame. The neutral atoms (large circles) move at the velocity-V relative to plasma. When ionized (the circle with + sign) their motion becomes affected by the magnetic field B carried with the plasma flow. The Figure shows the simplest case where the B field is perpendicular to the velocity V. The ions motion in the plasma frame immediately after pick-up consists then of rotation (with the original speed V) around the magnetic field direction. If the B field is inclined, the ions motion is composed of rotation and sliding along the magnetic field. Soon after the pick-up, scattering off magnetic field irregularities causes the ion velocity distribution to become partly isotropic ("shell" distribution).

\subsection{Evidence for Collisional Evolution near the Sun}

Evidence for the collisional evolution in the solar system dust cloud can be found in the minor species of the interplanetary medium plasma, namely in the pick-up ions that are produced when neutrals are ionized and then carried with the solar wind. Material released from dust particles by various mechanisms including vaporization, sublimation, desorption or direct collisions provide a source of neutral and ionized molecules, atoms and ions in the solar-wind plasma of the interplanetary medium. The neutral gas is quickly ionized by the solar wind and photons and picked up by the plasma of the solar wind. As illustrated in Figure 8, the freshly ionized particles start gyrating around the magnetic field that is carried in the solar wind plasma. As a consequence ions move outward with the solar wind. Since, at larger heliocentric distances, further ionization by the solar photons is unlikely and collisions with other species which could cause ionization are less frequent, the ions in majority keep their single charge state. Pick-up ions are distinguished from the solar-wind ions by their single charge state as well as by their velocity distribution (see Figure 9). These dust-related, or meteoritic production of ions gains importance in the inner solar system, where the number density and relative velocities of dust are the highest. These dust generated ions are proposed as an explanation of the inner source component of the pick-up ions discovered by Ulysses. The main component of pick-up ions in the solar wind is formed by the ionization of interstellar neutral gas that streams into the solar system. This inner source contains especially carbon, which is not a component of the interstellar neutral gas (Geiss, Gloeckler \& Steiger 1996). The inner source increases towards the Sun, which suggests it is correlated to the dust distri- 


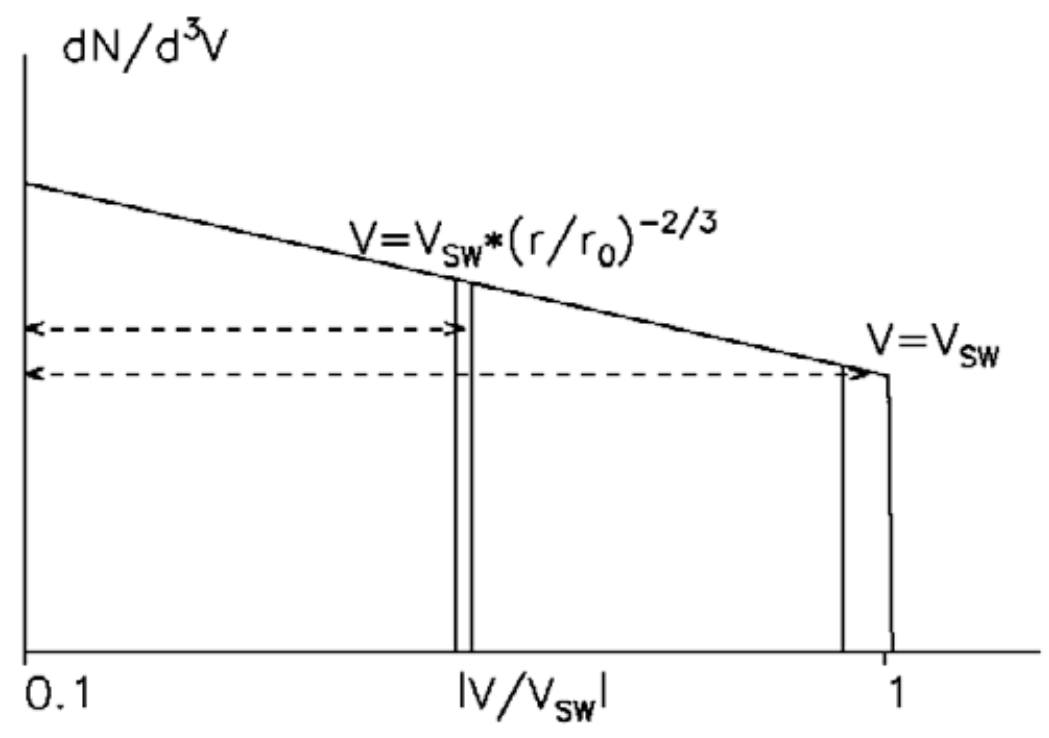

Figure 9. Pick-up ion velocity distribution at the distance $r$ from the Sun (velocity in the plasma frame). The distribution includes the ions picked up at all distances less than r. Freshly picked ions have the speed $|V|$ equal to the solar wind speed $V_{S W}$. The ions picked up previously (at the distance $\left.r_{0}<r\right)$ appear with reduced speed $\left(V=V_{S W} *\left(r / r_{0}\right)^{-2 / 3}\right.$ for isotropic pick-up ion velocity distribution and constant solar wind speed $\left.V_{S W}\right)$ because of adiabatic cooling caused by plasma expansion (solar wind density decreases as $1 / r^{2}$ ).

bution (Geiss, Gloeckler \& Steiger 1996; Gloeckler \& Geiss 1998, 2001). Noble gases and light elements in the inner source pick-up ions have abundances similar to that of the slow solar wind. Molecular ions in the mass range up to $40 \mathrm{amu}$ have also been detected (Gloeckler \& Geiss 2001). The similarity to solar-wind abundances suggests desorption of the solar-wind constituents is an important mechanism for the origin of these pick-up ions. However, the fluxes of dust required to account for the amounts of observed pick-up ions exceed by orders of magnitude the fluxes deduced from Zodiacal light observations (Mann, Kimura, Biesecker et al. 2004) and therefore surface interactions on the dust can not explain the observed ions.

In a recent study Mann \& Czechowski (2005) have shown that collisional vaporization of dust and meteoroids can account for the observed fluxes of heavy inner source pick-up ions and that the ion production from this process exceeds the production from other dust-related processes (see Figure 10). (Aside from the collisional destruction, significant amounts of ions are produced by dust sublimation near the Sun and since sublimation takes place in the most inner regions, the produced ions are multiply charged.)

The observed fraction of carbon among the pick-up ions cannot be explained by the fragmentation of materials with meteoritic element abundances, but rather with cometary dust. The carbon content for carbonaceous chondrites as the most primitive meteorite material is clearly below the values for cometary dust at comet Halley. This agrees with studies of the near solar dust cloud that have shown that it is most likely locally resupplied by collisional fragmentation of cometary meteoroids. The model of the pick-up ion production from dust cannot fully explain the present few data, but the detection of inner source ions implies that cometary dust contains carbon bearing species that can survive high temperatures in the vicinity of the Sun (Mann \& Czechowski 2005). 


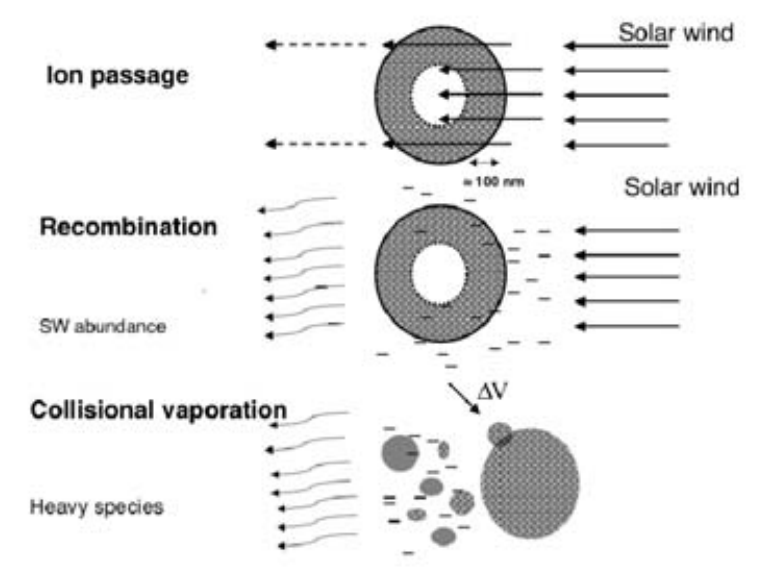

Figure 10. Dust interactions with the solar wind plasma of the interplanetary medium and the calculated amount of ions released by these processes for a typical model of dusta densities in the inner solar system. The collisional destruction provided the largest source of ions (Mann \& Czechowski 2005)

Nevertheless, it is not possible at present to directly derive dust compositions from the pick-up-ion measurements. The charge states of the ions depend on the distance from the Sun at which they are released as well as on the actual solar-wind conditions at that time. For ions released inward from 0.1 AU of the Sun, multiple ionization frequently occurs, so that depending on the atomic species and the solar-wind condition only about half of the total ions appear in the singly charged state.

\section{Summary}

The overall structure of the solar system dust cloud results from the contribution of asteroids, comets, Kuiper Belt objects, and interstellar dust. The two latter components produce the dust seen outward from about $5 \mathrm{AU}$ and are less important in the inner solar system. The dust near Earth orbit results mainly from asteroids and comets. Estimates of their relative contributions vary, which is partly a result of the different detection techniques, many of which are biased to the dust produced from asteroids. Dust from asteroids has typically smaller velocities relative to Earth and hence larger survival probability and therefore is overabundant in collected samples. Dust from asteroids also has a higher albedo and therefore more contributes to the Zodiacal light. This bias may not occur for meteors and indeed the orbital distribution of the sporadic meteors is different from orbital distributions derived from Zodiacal light models. Consideration of the collisional evolution in the dust cloud show that local sources need to replenish the cloud inward from $1 \mathrm{AU}$, and that cometary meteoroids are a likely source. Collision processes and dust sublimation feed ions into the solar wind plasma and it is expected that these species make up the heavy inner source pick-up ions that are seen in solar wind measurements.

Of the different constituents of the solar system dust cloud, the cometary dust undergoes the most complex alteration. Most of the alteration takes place in the coma and 
is connected to the evolution of the gas component. Fragmentation of dust in the coma is inferred from Stardust measurements as well as from brightness observations of the cometary dust. The fragmentation process is unclear but may include dust collisions, sublimation of volatiles and other effects. The break-up of larger particles due to sublimation of ice inclusions seems particularly plausible and would explain the irregular dust fluxes. All these processes may provide a source of coma gas, but yet carbon-bearing species will remain in the dust component. Particularly the CHON component detected with in-situ experiments of cometary dust is refractory and does not account for extended gas sources in the coma. The mass spectra obtained from in-situ measurements show no indication that the $\mathrm{CHON}$ component changes with distance from the comet, and particles were measured at distances from the nucleus where ices were sublimated already.

Both the optical properties of dust particles and the laboratory analyses of IDPs of likely cometary origin point to the fact that cometary dust is porous and irregular in shape, with the size of single monomers of the order of $100 \mathrm{~nm}$. The low albedo of the cometary dust, however, results from darkness of the material forming the single monomers rather than from the fluffy structure. These properties are best explained when optically dark components, possibly organic refractories, are a constituent of the single monomers. This cometary dust model is consistent with the model assumption that the interstellar grains incorporated into the cometary nuclei form large aggregates of core-mantle particles. Neverthess, this similarity should be viewed with some caution, since it is plausible to assume that only a fraction of the dust in the solar nebula may survive and be built directly into the cometary nuclei. Processing of the dust will occur prior to, during and after incorporation into the cometary nucleus.

The models of optical properties, the detection of carbon in the inner source pick-up ions generated by cometary meteoroids as well as the in-situ measurements of dust at comet Halley indicate that carbon-bearing species are intimately related to the silicate component of the cometary dust. We expect that a considerable fraction of the organic materials that are assumed to be present in the cometary dust formed refractory species. Traces of these remnants of the organic refractories are possibly seen in the ion composition of the solar wind.

\section{Acknowledgements}

We thank Dr. David Jewitt, Dr. George Flynn, and Dr. Masateru Ishiguro for helpful discussions and Dr. Diane Wooden for the careful review of the manuscript. Part of this review results from collaboration during I.M.'s and T.M.'s stay at the Institute of Low Temperature Science, Hokkaido University, the financial support for this stay is acknowledged. This research has been supported by the German Aerospace Center, DLR (project 'Rosetta: MIDAS, MIRO, MUPUS' RD-RX-50 QP 0403) and by the Japanese Ministry of Education, Culture, Sports, Science and Technology, MEXT, (Monbu Kagaku Sho) under Grant-in-Aid for Scientific Research on Priority Areas "Development of ExtraSolar Planetary Science" (16077203).

\section{References}

Baggaley, W.J. \& Neslusan, L. 2002, A\&A 382, 1118

Baum, W.A., Kreidl, T.J., \& Schleicher, D.G. 1992, AJ 104, 1216

Becklin, E.E. \& Westphal, J.A. 1966, ApJ 145, 445

Bertaux, J.L. \& Blamont, J.E. 1976, Nature 262, 263

Bockelée-Morvan, D. \& Crovisier, J. 2002, Earth Moon $\&$ Planets 89, 53

Bockelée-Morvan, D., Crovisier, J., Mumma, M.J., \& Weaver, H.A. 2005, in: M. Festou, H.U. Keller, \& H.A. Weaver (eds.) Comets II (Tucson: University of Arizona Press), p. 391 
Bradley, J.P., Brownlee, D.E., \& Fraundorf, P. 1984, Science 226, 1432

Bradley, J.P. 1988, Geochimica et Cosmochimica Acta 52, 889

Bradley, J.P. 1994, Science 265, 925

Bradley, J.P., Keller, L.P., Snow, T.P., Hanner, M.S., Flynn, G.J., Gezo, J.C., Clemett, S.J., Brownlee, D.E., \& Bowey, J.E. 1999, Science 285, 1716

Brownlee, D.E. 1978, in: J.A.M. McDonnell (ed.), Cosmic Dust (Wiley-Interscience) p. 295

Brownlee, D.E. 1985, in: Properties and interactions of interplanetary dust; Proceedings of the Eighty-fifth Colloquium (D. Reidel Publishing Co.) p. 143

Campins, H. \& Ryan, E.V. 1989, ApJ 341, 1059

Ceplecha, Z., Borovicka, J., Elford, W.G., Revelle, D.O., Hawkes, R.L., Porubčan, V., \& Šimek, M. 1998, Space Sci. Rev. 84, 327

Clark, B.C., Green, S.F., Economou, T.E., Sandford, S.A., Zolensky, M.E., McBride, N., \& Brownlee, D.E. 2004, J. Geophys. Res. 109, Issue E12, CiteID E12S03

Combi, M.R. 1994, AJ 108, 304

Combi, M.R., Kabin, K., DeZeeuw, D.L., Gombosi, T.I., \& Powell, K.G. 1997, Earth, Moon, \& Planets 79, 275

Crifo, J.F. 1995, ApJ 445, 470-488

Crifo, J.F. \& Rodionov, A.V. 1999, Planet. Space Sci. 47, 797

Disanti, M.A., Mumma, M.J., dello Russo, N., Magee-Sauer, K., Novak, R., \& Rettig, T.W. 1999, Nature 399, 662

Disanti, M.A., Mumma, M.J., \& dello Russo, N. 2001, Icarus 153, 361

Dobrovolsky, O.V., Kiselev, N.N., \& Chernova, G.P. 1986, Earth, Moon 6 Planets 34, 189

Dollfus, A., Bastien, P., Le Borgne, J.-F., Levasseur-Regourd, A.C., \& Mukai, T. 1988, A\&A 206,348

Draine, B.T. 1988, ApJ 333, 848

Draine, B.T. \& Flatau, P.J. 1994, Journal of the Optical Society of America A A11(4), 1491

Eberhardt, P. 1999, Space Sci. Rev. 90, 45

Fechtig, H. 1982, in: H. Wilkening (ed.) Comets (Tucson: University of Arizona Press) p. 383

Festou, M.C. 1999, Space Sci. Rev. 90, 53

Flynn, G.J. 1989, Icarus 77, 287

Flynn, G.J. 1996, in: B.A.S. Gustafson \& M.S. Hanner (eds.) Astronomical Society of the Pacific Conference Series; Proceedings of the 150th colloquium of the International Astronomical Union ASP 104, p. 171

Flynn, G.J., Keller, L.P., Feser, M., Wirick, S., \& Jacobsen, C. 2003, Geochimica et Cosmochimica Acta 67, 4791

Fomenkova, M. \& Chang, S. 1997, in: J.M. Greenberg (ed.) Proceedings of the NATO Advanced Study Institute (The Cosmic Dust Connection) 487, 459

Frisch, P.C., Dorschner, J.M., Geiss, J., Greenberg, J.M., Grünn, E., Landgraf, M., Hoppe, P., Jones, A.P., Krtschmer, W., Linde, T.J., Morfill, G.E., Reach, W., Slavin, J.D., Svestka, J., Witt, A.N., \& Zank, G.P. 1999, ApJ 525, 492

Furusho, R., Suzuki, B., Yamamoto, N., Kawakita, H., Sasaki, T., Shimizu, Y., \& Kurakami, T. 1999, PASJ 51, 367

Geiss, J., Gloeckler, G., \& von Steiger, R. 1996, Space Sci. Rev. 78, 43

Gloeckler, G., Fisk, L.A., Geiss, J., Schwadron, N.A., \& Zurbuchen, T.H. 2000, Journal of Geophysical Research 105, A4, 7459

Gloeckler, G. \& Geiss, J. 2001, Space Sci. Rev. 97, 169

Green, S.F., McDonnell, J.A.M., McBride, N., Colwell, M.T.S.H., Tuzzolino, A.J., Economou, T.E., Tsou, P., Clark, B.C., \& Brownlee, D.E. 2004, J. Geophys. Res. 109, Issue E12, CiteID7E12S04

Greenberg, J.M. \& Hage, J.I. 1990, ApJ 361, 260

Greenberg, J.M. \& Li, A. 1998, A\& $A$ 332, 374

Grün, E., Pailer, N., Fechtig, H., \& Kissel, J. 1980, Planet. Space Sci. 28, 333

Grün, E., Zook, H.A., Fechtig, H., \& Giese, R.H. 1998, Icarus 62, 244 
Grün, E., Gustafson, B., Mann, I., Baguhl, M., Morfill, G.E., Staubach, P., Taylor, A., \& Zook, H.A. 1994, A\&A 286, 915

Hadamcik, E. \& Levasseur-Regourd, A.C. 2003, A\& A 403, 757

Hadamcik, E., Renard, J., Levasseur-Regourd, A.C., \& Worms, J.C. 2003, JQSRT 79, 679

Hajduk, A. 2001, in: B. Warmbein (ed.) Meteoroids 2001 (ESA Publications Division, Noordwijk) ESA SP-495, p. 557

Hanner, M.S., Lynch, D.K., \& Russell, R.W. 1994, ApJ 425, 247

Hanner, M.S., Gehrz, R.D., Harker, D.E., Hayward, T.L., Lynch, D.K., Mason, C.C., Russell, R.W., Williams, D.M., Wooden, D.H., \& Woodward, C.E. 1997, Earth, Moon \& Planets 79,247

Hanner, M.S. 1999, Space Sci. Rev. 90, 998

Hanner, M.S. 2003, in: T.K. Henning (ed.) Astromineralogy (Springer) p. 609

Hanner, M.S. \& Bradley, J.P. 2005, in: M. Festou, H.U. Keller, \& H.A. Weaver (eds.) Comets II (Tucson: University of Arizona Press), p.

Harker, D.E., Wooden, D.H., Woodward, C.E., \& Lisse, C.M. 2002, ApJ 580, 579

Harker, D.E., Wooden, D.H., Woodward, C.E., \& Lisse, C.M. 2004, ApJ 615, 1081

Hasegawa, H., Ichikawa, T., Abe, S., Hamamura, S., Ohnishi, K., \& Watanabe, J. 1999, Earth, Moon, \& Planets 78, 353

Hübner, W.F. \& Benkhoff,, J. 1999, Space Sci. Rev. 90, 117

Hübner, W.F. 2002, Earth, Moon, \& Planets 89, 179

Hudson, B., Flynn, G.J., Fraundorf, P., Hohenberg, C.M., \& Shirck, J. 1981, Science 211, 383

Ishiguro, M., Watanabe, J., Usui, F., Tanigawa, T., Kinoshita, D., Suzuki, J., Nakamura, R., Ueno, M., \& Mukai, T. 2002, ApJ 572, L117

Ishimoto, H. \& Mann, I. 1999, Planet. Space Sci. 47, 225

Ishimoto, H. 2000, A E A 362, 1158

Janches, D., Pellinen-Wannberg, A., Wannberg, G., Westman, A., Häggström, I., \& Meisel, D.D. 2002, J. Geophys. Res. 107, A11, 14-1

Jessberger, E.K., Christoforidis, A., \& Kissel, J. 1988, Nature 332, 691

Jessberger, E.K., Stephan, T., Rost, D., Arndt, P., Maetz, M., Stadermann, F.J., Brownlee, D.E., Bradley, J.P., \& Kurat, G. 2001, in: E. Grün, B.A.S. Gustafson, S.F. Dermott, \& H. Fechtig (eds.) Interplanetary Dust (Springer-Verlag) p. 253

Jewitt, D. 2004, AJ 128, 3061

Jockers, K., Rosenbush, V., Bonev, T., \& Credner, T. 1999, Earth, Moon, \&3 Planets 78, 373

Jockers, K., Kiselev, N., Bonev, T., Rosenbush, V., Shakhovskov, N., Kolesnikov, S., Efimov, Y., Shakhovskoy, D., \& Antonyuk, K. 2005, A\&\&A 441, 773

Joswiak, D.J., Brownlee, D.E., Bradley, J.P., Schlutter, D.J., \& Pepin, R.O. 1996, Lunar and Planetary Institute Conference Abstracts, p. 625

Keller, L.P., Messenger, S., Flynn, G.J., Wirick, S., \& Jacobsen, C. 2002, Meteoritics 8 Planetary Sci. 37,76

Kelley, M.S., Woodward, C.E., Jones, T.J., Reach, W.T., \& Johnson, J. 2004, AJ 127, 2398

Kimura, H., Mann, I., \& Mukai, T. 1998, Planet. Space Sci. 46, 911

Kimura, H., Kolokolova, L., \& Mann, I. 2003, A\&A 407, L5

Kimura, H., Mann, I., \& Jessberger, E.K. 2003, Icarus ApJ, 314

Kiselev, N.N. \& Velichko, F.P. 1998, Icarus 133, 286

Kiselev, N.N. \& Velichko, F.P. 1999, Earth, Moon, \& Planets 78, 347

Kissel, J., Brownlee, D.E., Büchler, K., Clark, B.C., Fechtig, H., Grün, E., Hornung, K., Igenbergs, E.B., Jessberger, E.K., Krueger, F.R., Kuczera, H., McDonnell, J.A.M., Morfill, G.M., Rahe, J., Schwehm, G.H., Sekanina, Z., Utterback, N.G., Völk, H.J., \& Zook, H.A. 1986, Nature 321, 336

Kissel, J., Sagdeev, R.Z., Bertaux, J.L., Angarov, V.N., Audouze, J., Blamont, J.E., Büchler, K., Evlanov, E.N., Fechtig, H., Fomenkova, M.N., von Hoerner, H., Inogamov, N.A., Khromov, V.N., Knabe, W., Krueger, F.R., Langevin, Y., Leonas, V.B., Levasseur-Regourd, A.C., Managadze, G.G., Podkolzin, S.N., Shapiro, V.D., Tabaldyev, S.R., \& Zubkov, B.V. 1986, Nature 321, 280 
Kneissel, B. \& Mann, I. 1991, in: A.C. Levasseur-Regourd \& H. Hasegawa (eds.) Origin and Evolution of Interplanetary Dust (Dordrecht: Kluwer) p. 131

Kolokolova, L., Kimura, H., \& Mann, I. 2004, in: G. Videen, Y. Yatskiv, \& M. Mishchenko (eds.) Photopolarimetry in Remote Sensing (Kluwer Academic Publisher), p. 431

Kolokolova, L., Jockers, K., Gustafson, B., \& Lichtenberg, G. 2001, J. Geophys. Res. 106, 10113

Kolokolova, L., Hanner, M.S., Levasseur-Regourd, A.-C., \& Gustafson, B.A.S. 2005, in: M. Festou, H.U. Keller, \& H.A. Weaver (eds.) Comets II (University of Arizona Press), p.

Konno, I., Hübner, W.F., \& Boice, D.C. 1993, Icarus 101, 84

Kurat, G., Koeberl, C., Presper, T., Brandsttter, F., \& Maurette, M. 1994, Geochimica et Cosmochimica Acta 58, Issue 18, 3879

Landgraf, M. 2000, J. Geophys. Res. 105, 10303

Lara, L.M., Schulz, R., Stüwe, J.A., \& Tozzi, G.P. 2001, Icarus 150, 124

Lawler, M.E. \& Brownlee, D.E. 1992, Nature 359, 810

Lederer, S.M., Osip, D.J., Thomas-Osip, J.E., DeBuizer, J.M., Mondragon, L.A., Schweiger, D.L., \& Viehweg, J., SB Collaboration 2005, AAS/Division for Planetary Sciences Meeting Abstracts 37, 4308

Levasseur-Regourd, A.C., Hadamcik, E., \& Renard, J. 1996, A\&A 313, 327

Levasseur-Regourd, A.C., Mann, I., Dumont, R., \& Hanner, M.S 2001, in: E. Grün, B.A.S. Gustafson, S.F. Dermott, \& H. Fechtig (eds.) Interplanetary Dust (Springer-Verlag) p. 57

Liou, J.-C., Zook, H.A., \& Dermott, S.F. 1996, Icarus 124, 429

Lumme, K. \& Rahola, J. 1994, ApJ 425, 653

Mann, I., Okamoto, H., Mukai, T., Kimura, H., \& Kitada, Y. 1994, A\&A 291, 1011

Mann, I. 1996, Space Sci. Rev. 78, 259

Mann, I. 1998, Earth, Planets, \& Space 50(6,7), 465

Mann, I. \& Kimura, H. 2000, J. Geophys. Res. 105, 10317

Mann, I. \& Kimura, H. 2001, Space Sci. Rev. 97, Issue 1/4, 389

Mann, I. \& Jessberger, E.K. 2003, in: T. Henning (ed.) Astromineralogy (Berlin: Springer) p. 98

Mann, I., Kimura, H., Biesecker, D.A., Tsurutani, B.T., Grün, E., McKibben, B., Liou, J.C., MacQueen, R.M., Mukai, T., Guhartakuta, L., \& Lamy, P. 2004, Space Sci. Rev. 110, 269

Mann, I., Kimura, H., \& Kolokolova, L. 2004, JQSRT 89, 291

Mann, I. \& Czechowski, A. 2005, ApJ 621, L73

Manset, N. \& Bastien, P. 2000, Icarus 145, 203

Maurette, M., Hammer, C., Reeh, N., Brownlee, D.E., \& Thomsen, H.H. 1986, Science 233, 869

Maurette, M., Olinger, C., Michel-Levy, M.C., Kurat, G., Pourchet, M., Brandstatter, F., \& Bourot-Denise, M. 1991, Nature 351, 44

Meisel, D.D., Janches, D., \& Mathews, J.D. 2002, ApJ 567, 323

Messenger, S. 2000, Nature 404, 968

Mukai, T., Mukai, S., \& Kikuchi, S. 1987, A\& A 187, 650

Mukai, T. \& Koike, C. 1990, Icarus 87, 180

Mukai, T., Ishiguro, M., \& Usui, F. 2002, Adv. Space Res. in press.

Ohgaito, R., Mann, I., Kuhn, J.R., MacQueen, R.M., \& Kimura, H. 2002, ApJ 578, 610

Pellinen-Wannberg, A. \& Wannberg, G. 1994, J. Geophys. Res. 99, 11397

Petrova, E.V., Jockers, K., \& Kiselev, N.N. 2000, Icarus 148, 526

Reach, W.T., Morris, P., Boulanger, F., \& Okumura, K. 2003, Icarus 164, 384

Rietmeijer, F.J.M. 1993, J. Geophys. Res. 98, 7409

Rietmeijer, F.J.M. 1999, in: J.J. Papike (ed.) Planetary Materials (Mineralogical Society of America), vol. 36. p. 2-1-2-95

Rietmeijer, F.J.M. 2000, Meteoritics $\&$ Planetary Sci. 35, 1025

Rietmeijer, F.J.M. 2002, in: E. Murad \& I.P. Williams (eds.) Meteors in the Earth's atmosphere (Cambridge: Cambridge University Press), p. 215

Schulze, H., Kissel, J., \& Jessberger, E.K. 2002, ASP Conf. Ser. 122: From Stardust to Planetesimals, p. 397

Schleicher, D.G. \& Osip, D.J. 2002, Icarus 159, 210

Sekanina, Z., Brownlee, D.E., Economou, T.E., Tuzzolino, A.J., \& Green, S.F. 2004, Science 304, 1769 
Sykes, M.V., Lebofsky, L.A., Hunten, D.M., \& Low, F. 1986, Science 1986, 1115

Sykes, M.V. \& Walker, R.G. 1992, Icarus 1992, 180

Taylor, A.D., Baggaley, W.J., \& Steel, D.I. 1996, Nature 380, 325

Tsou, P., Brownlee, D.E., Anderson, J.D., Bhaskaran, S., Cheuvront, A.R., Clark, B.C., Duxbury, T., Economou, T., Green, S.F., Hanner, M.S., Hörz, F., Kissel, J., McDonnell, J.A.M., Newburn, R.L., Ryan, R.E., Sandford, S.A., Sekanina, Z., Tuzzolino, A.J., Vellinga, J.M., \& Zolensky, M.E. 2004, J. Geophys. Res. 109,

Tsurutani, B.T.et al. 2004, Icarus 167, 89

Turner, N.J. \& Smith, G.H. 1999, ApJ 118, 3039

Tuzzolino, A.J., Economou, T.E., Clark, B.C., Tsou, P., Brownlee, D.E., Green, S.F., McDonnell, J.A.M., McBride, N., \& Colwell, M.T.S.H. 2004, Science 304, 17760

Wooden, D.H., Harker, D.E., Woodward, C.E., Butner, H.M., Koike, C., Witteborn, F.C., \& McMurtry, C.W. 2004, ApJ 517, 1034

Wooden, D.H., Harker, D.E., \& Woodward, C.E. 2000, ASP Conf. Ser. 196: Thermal Emission Spectroscopy and Analysis of Dust, Disks, and Regoliths 196, 99

Wooden, D.H. 2002, Earth Moon 8 Planets 89, 247

Wooden, D.H., Charnley, S.B., \& Ehrenfreund, P. 2005, in: M. Festou, H.U. Keller \& H.A. Weaver (eds.) Comets II (Tucson: University of Arizona Press) p.

Woodward, C.E., Shure, M.A., Forrest, W.J., Jones, T.J., Gehrz, R.D., Nagata, T., \& Tokunaga, A.T. 1996, Icarus 124, 651 